\title{
ABSORÇÃO DE PESADOS DO GÁS NATURAL UTILIZANDO SIMULAÇÃO COMPUTACIONAL
}

\author{
D. N. N. da SILVA ${ }^{1}$, O. CHIAVONE FILHO ${ }^{1}$ \\ ${ }^{1}$ Universidade Federal do Rio Grande do Norte, Departamento de Engenharia Química \\ E-mail para contato: daniel.eq@hotmail.com
}

\begin{abstract}
RESUMO - O objetivo deste projeto é determinar qual absorvente é o mais eficiente na remoção dos compostos pesados $(\mathrm{C} 3+)$ do gás natural, a partir de simulações computacionais. Inicialmente foi identificado qual dos modelos termodinâmicos em estudo promoveu menores erros se comparados aos dados experimentais. Foram verificados diversos absorventes, tais quais, etanoato de etila, 1-butanol e 1-octanol. Utilizando o software Specs ${ }^{\circledR}$ e diversos combinações de modelos, SRK e Peng Robinson, e regra de mistura QMR. Foi utilizado como parâmetro principal a pressão de bolha. Realizado as diversas simulações foi identificado os modelos que mais se ajustaram para cada absorvente utilizado. Realizando simulações de separação flash no mesmo software foi identificado que o absorvente 1-octanol obteve melhor resultado na remoção destes pesados além de promover baixa concentração de absorvente no gás processado.
\end{abstract}

\section{INTRODUÇÃO}

A utilização do gás natural vem crescendo ano após ano em todo o mundo e também no Brasil. Verifica-se que nos últimos cinco anos o perfil de consumo de gás natural veicular ganhou um grande alcance e vários investimentos foram realizados nesta área. Na indústria do petróleo a utilização do gás natural para combustível no acionamento de motores já vem de longa data, e o mesmo é utilizado também para movimentar equipamentos, ou ainda, para gerar energia elétrica.

A remoção dos componentes mais pesados $(\mathrm{C} 3+)$ é necessária para adequar esse gás natural às especificações requeridas para uma queima adequada e otimizada. Utilizando dados experimentais avaliamos os diversos modelos e avaliamos qual obteve menores erros e com isso utilizar esse modelo para simular as separações flash, encontrando o melhor absorvente.

\section{ASPECTOS TEÓRICOS}

\subsection{Gás Natural}

O gás natural consiste numa mistura de hidrocarbonetos leves e gases inorgânicos saturados com água. Em alguns casos pode conter traços de mercúrio em equilíbrio. O gás associado é geralmente produzido a baixas pressões, visto que isto maximiza a produção de hidrocarbonetos de determinado reservatório - óleo e gás. Assim sendo, por estar associado ao óleo, o gás associado possui quantidades significativas de hidrocarbonetos pesados, que conferem um maior peso molecular e consequentemente uma maior massa específica. $\mathrm{O}$ gás 
não associado por sua vez possui uma sequência de hidrocarbonetos constituintes mais leves, entretanto pode ser produzido a pressões mais altas, o que pode ser uma vantagem nas etapas de transporte e processamento.

\subsection{Cálculos de Propriedades Termodinâmicas}

O cálculo das propriedades termodinâmicas para hidrocarbonetos leves a altas pressões e soluções que envolvem equilíbrio com estas substâncias podem ser desenvolvidas com base em equações de estado (EDE) (Sandler, 1994).

Os modelos abordados neste trabalho que são baseados em EDE são:

- Soave-Redlich-Kwong (1972);

- Peng-Robinson (1976);

São mostradas a seguir, as equações básicas para modelagem termodinâmica a partir dos estudos de Peng-Robinson:

$$
\begin{aligned}
& P=\frac{R T}{\underline{V}-b}-\frac{a(T)}{\underline{V}(\underline{V}+b)+b(\underline{V}-b)} \\
& a(T)=0,45724 \frac{R^{2} T_{C}^{2}}{P_{C}} \alpha(T) \\
& b=0,07780 \frac{R T_{C}}{P_{C}} \\
& {[\alpha(T)]^{\frac{1}{2}}=1+\kappa\left(1-\sqrt{\frac{T}{T_{C}}}\right)} \\
& \kappa=0,37464+1,5422 \omega-0,26992 \omega^{2}
\end{aligned}
$$

Onde $P$ é a pressão do ponto de bolha, $R$ é a constante real dos gases ideais, $\underline{V}$ é o volume molar do componente puro, $T$ é a temperatura em que se encontra o componente, $T_{C}$ é a temperatura critica desse componente e $\omega$ é o fator acêntrico.

Os parâmetros apresentados pelas Equações 2 e 3 são para componentes puros, então devemos utilizar uma regra de mistura para obtermos os parâmetros $a(T)$ e $b$. Neste trabalho iremos usar dois tipos de regra de mistura, para o modelo baseado na EDE proposto por PengRobinson usamos um desses dois tipos, a regra de mistura quadrática (Quadratic Mixing Rule - QMR). As equações que descrevem a QMR são mostradas a seguir:

$$
\begin{aligned}
a & =\sum_{i=1}^{c} \sum_{j=1}^{c} x_{i} x_{j} a_{i j} \\
b & =\sum_{i=1}^{c} \sum_{j=1}^{c} x_{i} x_{j} b_{i j}
\end{aligned}
$$




$$
\begin{aligned}
& a_{i j}=\sqrt{a_{i i} a_{j j}}\left(1-k_{i j}\right)=a_{j i} \\
& b_{i j}=\frac{1}{2}\left(b_{i i}+b_{j j}\right)\left(1-l_{i j}\right)
\end{aligned}
$$

Os parâmetros $a_{i i}, b_{i i}, b_{j j}$ e $a_{j j}$ são parâmetros para o componente puro $i$ e $j$. Os parâmetros $k_{i j}$ e $l_{i j}$ são conhecidos como parâmetros de interação binária, e são encontrados por um ajuste da equação de estado para dados de equilíbrio líquido-vapor da mistura. Geralmente $l_{i j}$ é igual à zero, logo a Equação 7 se tornará:

$$
\begin{aligned}
& b_{i j}=\frac{1}{2}\left(b_{i i}+b_{j j}\right)=b_{i i} \\
& b=\sum_{i=1}^{c} x_{i} b_{i i}
\end{aligned}
$$

Agora são mostradas a seguir as equações básicas para modelagem termodinâmica da EDE Soave-Redlich-Kwong (SRK):

$$
\begin{aligned}
& P=\frac{R T}{\underline{V}-b}-\frac{a(T)}{\underline{V}(\underline{V}+b)} \\
& a(T)=a\left(T_{C}\right) \alpha(T) \\
& a\left(T_{C}\right)=0,42748 \frac{\left(R T_{C}\right)^{2}}{P_{C}} \\
& \alpha(T)=\left[1+\left(0,480+1,574 \omega-0,176 \omega^{2}\right)\left(1-\sqrt{T / T_{C}}\right)\right]^{2} \\
& b=0,08664 \frac{R T_{C}}{P_{C}}
\end{aligned}
$$

\section{METODOLOGIA}

Foram utilizados dados de equilíbrio liquido-vapor da literatura para avaliar os modelos utilizados no trabalho através do software SPECS ${ }^{\circledR}$. Os testes foram realizados em três absorventes, etanoato de etila, 1-butanol e 1-octanol. Para cada foi realizado diversas simulações da etapa de absorção onde se retira a maior parte das impurezas do gás natural bruto.

Foram utilizados dois modelos termodinâmicos, o SRK + QMR e PR + QMR, onde o parâmetro de interação binaria da regra de mistura $k_{i j}$ é considerado diferentes de zero. Para o processo de simulação teremos um processo flash no software SPEC $^{\circledR}$, onde o gás bruto e o absorvente em questão entram juntos na mesma entrada e são separados em duas correntes, uma liquida (absorvente contaminado) e uma gasosa (gás natural processado). 
Tabela 1 - Composições típicas do gás natural

\begin{tabular}{|c|c|c|}
\hline Componentes & Gás associado (molar) & Gás Processado (molar) \\
\hline Metano & 0,692 & 0,862 \\
\hline Etano & 0,106 & 0,095 \\
\hline Propano & 0,074 & 0,010 \\
\hline Butanos & 0,043 & 0,000 \\
\hline Pentanos & 0,021 & 0,000 \\
\hline Hexanos & 0,0106 & 0,000 \\
\hline Heptanos & 0,0085 & 0,000 \\
\hline Gás carbônico & 0,0133 & 0,013 \\
\hline Nitrogênio & 0,024 & 0,014 \\
\hline Água & 0,007 & 0,007 \\
\hline
\end{tabular}

Fonte: Petrobras

Para avaliarmos se os modelos utilizados predizem de forma satisfatória os dados experimentais, utilizamos os sistemas abaixo apresentados na Tabela 2.

Tabela 2 - Sistemas de absorventes para estudo de predição

\begin{tabular}{|c|}
\hline Sistemas \\
\hline n-Butano (x1)+ Etanoato de Etila (x2) \\
\hline n-Butano (x1)+ 1-Butanol (x2) \\
\hline n-Heptano (x1)+ 1-Octanol (x2) \\
\hline
\end{tabular}

Utilizamos quatro composições diferentes de absorvente na corrente de entrada do flash, já a composição do gás será proporcional à composição do gás bruto com a consideração que teremos somente pesados até o heptano. Temos que as composições são apresentadas na Tabela 3.

Tabela 3 - Composição molar da corrente de entrada no flash de acordo com quantidade de absorvente

\begin{tabular}{|c|c|c|c|}
\hline Componente & \multicolumn{3}{|c|}{ Composições } \\
\hline Absorvente & 0,1 & 0,3 & 0,5 \\
\hline Metano & 0,6224 & 0,4841 & 0,3458 \\
\hline Etano & 0,0957 & 0,0745 & 0,0532 \\
\hline Propano & 0,0670 & 0,0521 & 0,0372 \\
\hline Butano & 0,0383 & 0,0298 & 0,0213 \\
\hline Pentano & 0,0192 & 0,0149 & 0,0107 \\
\hline Hexano & 0,0095 & 0,0074 & 0,0053 \\
\hline Heptano & 0,0076 & 0,0059 & 0,0042 \\
\hline CO2 & 0,0120 & 0,0093 & 0,0067 \\
\hline Nitrogênio & 0,0215 & 0,0168 & 0,0120 \\
\hline H2O & 0,0067 & 0,0052 & 0,0037 \\
\hline
\end{tabular}




\section{RESULTADO E DISCUSSÕES}

Os dados experimentais de pressão de bolha dos sistemas apresentados na tabela 3 são mostrados nas Tabelas 4,5 e 6.

Tabela 4 - Dados experimentais para o sistema n-butano (1) + etanoato de etila (2)

\begin{tabular}{|c|c|c|c|c|}
\hline Ponto & $\mathrm{T}(\mathrm{K})$ & $\mathrm{x} 1$ & $\mathrm{x} 2$ & $\mathrm{P}(\mathrm{kPa})$ \\
\hline 1 & 318,4 & 0,897 & 0,103 & 402,95 \\
\hline 2 & 318,4 & 0,597 & 0,403 & 323,26 \\
\hline 3 & 318,4 & 0,351 & 0,649 & 246,76 \\
\hline 4 & 318,4 & 0,102 & 0,898 & 116,47 \\
\hline
\end{tabular}

Fonte: HAIMI, P. et al

Tabela 5 - Dados experimentais para o sistema n-butano (1) + 1-butanol (2)

\begin{tabular}{|c|c|c|c|c|}
\hline Ponto & $\mathrm{T}(\mathrm{K})$ & $\mathrm{x} 1$ & $\mathrm{x} 2$ & $\mathrm{P}(\mathrm{kPa})$ \\
\hline 1 & 333,22 & 0,4174 & 0,5826 & 491 \\
\hline 2 & 333,48 & 0,6072 & 0,3928 & 559 \\
\hline 3 & 333,25 & 0,8114 & 0,1886 & 598 \\
\hline
\end{tabular}

Fonte: DÉAK, A. et al.

Tabela 6 - Dados experimentais para o sistema n-heptano (1) + 1-octanol (2)

\begin{tabular}{|c|c|c|c|c|}
\hline Ponto & $\mathrm{T}(\mathrm{K})$ & $\mathrm{x} 1$ & $\mathrm{x} 2$ & $\mathrm{P}(\mathrm{kPa})$ \\
\hline 1 & 293,15 & 0,755 & 0,245 & 4,34 \\
\hline 2 & 293,15 & 0,983 & 0,017 & 4,68 \\
\hline 3 & 313,15 & 0,836 & 0,164 & 11,72 \\
\hline 4 & 313,15 & 0,965 & 0,035 & 12,19 \\
\hline 5 & 408,21 & 0,2 & 0,8 & 101,32 \\
\hline \multicolumn{5}{c}{ Fonte: GÓRAL, M. et al }
\end{tabular}

Utilizando estes dados podemos avaliar a aderência dos modelos utilizados para predizer o processo de separação do gás natural. Onde os erros médios de cada sistema para cada modelo são mostrados na Tabela 7.

Tabela 7 - Erros (\%) encontrados através das simulações para cada modelo

\begin{tabular}{|c|c|c|}
\hline Sistema & Erro \% - PR + QMR & Erro \% - SRK + QMR \\
\hline n-Butano (x1)+ Etanoato de Etila (x2) & 0,638 & 0,305 \\
\hline n-Butano (x1)+ 1-Butanol (x2) & 5,497 & 5,308 \\
\hline n-Heptano (x1)+ 1-Octanol (x2) & 3,09949 & 5,62898 \\
\hline
\end{tabular}

Onde temos que o erro é calculado da seguinte forma:

$$
\operatorname{Erro}(\%)=\frac{100}{N} \sum_{i=1}^{N}\left|\frac{P_{c a l c}-P_{\exp t l}}{P_{\exp t l}}\right|
$$


Após realização das simulações do processo de separação flash para os três absorventes em estudo obtivemos os seguintes resultados:

Absorvente etanoato de etila: Realizado as simulações de separação fash, observamos que não houve diferença considerável entre os dois modelos utilizados, desvio médio de 0,00102 na composição de metano na corrente de gás processado, logo iremos nós ater apenas a analisar os dados obtidos pelo modelo de $\mathrm{PR}+\mathrm{QMR}$ com $\mathrm{k}_{\mathrm{ij}}$ igual à zero. Devido à ausência de dados experimentais de equilíbrio liquido-vapor dos absorventes com cada componente do gás natural bruto ficou difícil calcular os $\mathrm{k}_{\mathrm{ij}}$ de todos os pares funcionais. Logo a adição somente dos $\mathrm{k}_{\mathrm{ij}}$ das espécies orgânica não foi observado mudança considerável entre os dados obtidos considerando $\mathrm{k}_{\mathrm{ij}}$ iguais a zero.

Temos que os resultados obtidos para o absorvente etanoato de etila são:

Tabela 8 - Composições das correntes de etanoato de etila contaminado e gás processado para cada quantidade de absorvente utilizado para o modelo $\mathrm{PR}+\mathrm{QMR}$ e $\mathrm{k}_{\mathrm{ij}}$ iguais à zero

\begin{tabular}{|c|c|c|c|c|c|c|}
\hline \multirow{2}{*}{ Componente } & \multicolumn{2}{|c|}{0,1 de absorvente } & \multicolumn{2}{c|}{0,3 de absorvente } & \multicolumn{2}{c|}{0,5 de absorvente } \\
\cline { 2 - 7 } & $\begin{array}{c}\text { Absorvente } \\
\text { cont. }\end{array}$ & $\begin{array}{c}\text { Gás } \\
\text { Proces. }\end{array}$ & $\begin{array}{c}\text { Absorvente } \\
\text { cont. }\end{array}$ & $\begin{array}{c}\text { Gás } \\
\text { Proces. }\end{array}$ & $\begin{array}{c}\text { Absorvente } \\
\text { cont. }\end{array}$ & $\begin{array}{c}\text { Gás } \\
\text { Proces. }\end{array}$ \\
\hline Metano & 0,01632 & 0,67884 & 0,01601 & 0,70079 & 0,01624 & 0,72383 \\
\hline Etano & 0,01278 & 0,10342 & 0,01235 & 0,10327 & 0,01189 & 0,10059 \\
\hline Propano & 0,02787 & 0,07065 & 0,0248 & 0,06474 & 0,02111 & 0,05565 \\
\hline Butano & 0,04665 & 0,03753 & 0,03382 & 0,02794 & 0,02309 & 0,01924 \\
\hline Pentano & 0,05736 & 0,01565 & 0,02932 & 0,00823 & 0,01606 & 0,00455 \\
\hline Hexano & 0,05487 & 0,00528 & 0,01926 & 0,00191 & 0,00912 & 0,00091 \\
\hline Heptano & 0,06475 & 0,00229 & 0,01728 & 0,00063 & 0,00762 & 0,00028 \\
\hline CO2 & 0,00127 & 0,013 & 0,00125 & 0,01303 & 0,00123 & 0,01297 \\
\hline Nitrogênio & 0,0002 & 0,02348 & 0,0002 & 0,02449 & 0,0002 & 0,02554 \\
\hline H2O & 0,0158 & 0,00585 & 0,00944 & 0,00324 & 0,00536 & 0,00179 \\
\hline Etanoato de Etila & 0,70214 & 0,044 & 0,8363 & 0,05174 & 0,88808 & 0,05465 \\
\hline
\end{tabular}

Absorvente 1-butanol: De forma análoga aos dados obtidos para o absorvente etanoato de etila, temos os resultados obtidos para o absorvente 1-butanol:

Tabela 9 - Composições das correntes de 1-butanol contaminado e gás processado para cada quantidade de absorvente utilizado para o modelo $\mathrm{PR}+\mathrm{QMR}$ e $\mathrm{k}_{\mathrm{ij}}$ iguais à zero

\begin{tabular}{|c|c|c|c|c|c|c|}
\hline \multirow{2}{*}{ Componente } & \multicolumn{2}{|c|}{0,1 de absorvente } & \multicolumn{2}{c|}{0,3 de absorvente } & \multicolumn{2}{c|}{0,5 de absorvente } \\
\cline { 2 - 7 } & $\begin{array}{c}\text { Absorvente } \\
\text { cont. }\end{array}$ & $\begin{array}{c}\text { Gás } \\
\text { Proces. }\end{array}$ & $\begin{array}{c}\text { Absorvente } \\
\text { cont. }\end{array}$ & $\begin{array}{c}\text { Gás } \\
\text { Proces. }\end{array}$ & $\begin{array}{c}\text { Absorvente } \\
\text { cont. }\end{array}$ & $\begin{array}{c}\text { Gás } \\
\text { Proces. }\end{array}$ \\
\hline Metano & 0,01117 & 0,70604 & 0,01056 & 0,72742 & 0,01048 & 0,7489 \\
\hline Etano & 0,00975 & 0,10746 & 0,00913 & 0,10809 & 0,00874 & 0,10665 \\
\hline Propano & 0,02109 & 0,07329 & 0,01844 & 0,0694 & 0,01608 & 0,06259 \\
\hline Butano & 0,03471 & 0,0388 & 0,02598 & 0,03176 & 0,01897 & 0,0241 \\
\hline Pentano & 0,04083 & 0,01625 & 0,02358 & 0,01044 & 0,01415 & 0,00656 \\
\hline
\end{tabular}




\begin{tabular}{|c|c|c|c|c|c|c|}
\hline Hexano & 0,0377 & 0,00565 & 0,01638 & 0,00279 & 0,00845 & 0,00152 \\
\hline Heptano & 0,04403 & 0,00262 & 0,01532 & 0,00106 & 0,00725 & 0,00054 \\
\hline CO2 & 0,00112 & 0,01349 & 0,00108 & 0,01352 & 0,00106 & 0,01348 \\
\hline Nitrogênio & 0,00012 & 0,02443 & 0,00011 & 0,02538 & 0,00011 & 0,0263 \\
\hline H2O & 0,02189 & 0,00462 & 0,01118 & 0,00213 & 0,00588 & 0,00107 \\
\hline 1-Butanol & 0,77758 & 0,00737 & 0,86825 & 0,00802 & 0,90884 & 0,00832 \\
\hline
\end{tabular}

Absorvente 1-octanol: Para o absorvente 1-octanol temos os seguintes resultados de separação por flash:

Tabela 10 - Composições das correntes de 1-octanol contaminado e gás processado para cada quantidade de absorvente utilizado para o modelo $\mathrm{PR}+\mathrm{QMR}$ e $\mathrm{k}_{\mathrm{ij}}$ iguais à zero

\begin{tabular}{|c|c|c|c|c|c|c|}
\hline \multirow{2}{*}{ Componente } & \multicolumn{2}{|c|}{0,1 de absorvente } & \multicolumn{2}{|c|}{0,3 de absorvente } & \multicolumn{2}{c|}{0,5 de absorvente } \\
\cline { 2 - 7 } & $\begin{array}{c}\text { Absorvente } \\
\text { cont. }\end{array}$ & $\begin{array}{c}\text { Gás } \\
\text { Proces. }\end{array}$ & $\begin{array}{c}\text { Absorvente } \\
\text { cont. }\end{array}$ & $\begin{array}{c}\text { Gás } \\
\text { Proces. }\end{array}$ & $\begin{array}{c}\text { Absorvente } \\
\text { cont. }\end{array}$ & $\begin{array}{c}\text { Gás } \\
\text { Proces. }\end{array}$ \\
\hline Metano & 0,01536 & 0,71629 & 0,01547 & 0,74225 & 0,01582 & 0,76776 \\
\hline Etano & 0,01243 & 0,10858 & 0,01222 & 0,10881 & 0,01183 & 0,1061 \\
\hline Propano & 0,02703 & 0,07319 & 0,02445 & 0,06733 & 0,02093 & 0,058 \\
\hline Butano & 0,04395 & 0,03743 & 0,03264 & 0,02823 & 0,0226 & 0,01964 \\
\hline Pentano & 0,05004 & 0,01444 & 0,02738 & 0,00803 & 0,0155 & 0,00456 \\
\hline Hexano & 0,04276 & 0,00436 & 0,01753 & 0,00182 & 0,00873 & 0,00091 \\
\hline Heptano & 0,04567 & 0,00172 & 0,01553 & 0,0006 & 0,00726 & 0,00028 \\
\hline CO2 & 0,00127 & 0,01366 & 0,00126 & 0,01373 & 0,00125 & 0,01367 \\
\hline Nitrogênio & 0,00018 & 0,0248 & 0,00018 & 0,02595 & 0,00019 & 0,0271 \\
\hline H2O & 0,01609 & 0,00525 & 0,00932 & 0,00293 & 0,00531 & 0,00164 \\
\hline 1-Octanol & 0,74524 & 0,00029 & 0,84402 & 0,00032 & 0,89058 & 0,00034 \\
\hline
\end{tabular}

\section{CONCLUSÕES}

Através da análise dos dados obtidos chegamos as seguintes conclusões:

- Visto possuir em qualquer composição, na corrente de alimentação, maior composição de metano na corrente de gás tratado, o absorvente 1-octanol é o mais eficiente em remoção dos pesados do gás natural bruto;

- Além de possuir essa maior composição de metano, o 1-octanol promove uma menor contaminação do gás processado por absorvente se comparado pelos outros absorventes;

- Foram realizados testes para a composição de absorvente de 0,6 , mas devido as elevadas quantidades de absorventes empregadas para uma aumento de $2 \%$ na composição de metano na corrente de gás processado; 
- Os modelos utilizados foram muito similares em simular estes dados, mas o modelo PR + QMR obteve melhores resultados.

\section{REFERÊNCIAS BIBLIOGRÁFICAS}

- DÉAK, A. \& VICTOROV, A.I. \& LOSS, Th.W. High pressure VLE in alkanol + alkane mixtures. Experimental results for n-butane + ethanol, +1-propanol, + 1butanol systems and calculations with three EOS methods. Fluid Phase Equilibria, Delft, v. 107, 1995, pp. 277- 301.

- GOMES, Cícero Sena Moreira. Remoção de hidrocarbonetos pesados do gás natural visando o ajuste do índice de metano. 2007. 131 f. Dissertação (Mestrado) Curso de Engenharia Química, Departamento de Engenharia Química, Universidade Federal do Rio Grande do Norte, Natal, 2007.

- GÓRAL, M. et al. Recommended vapor-liquid equilibrium data. Part 1: Binary nAlkanol-n- Alkane systems. J.Physi.Chem.Ref.Data, Warsaw, v. 31,n.3, 2002, pp.701- 745 .

- HAIMI, P. et al, Vapour-liquid equilibrium for the ethyl ethanoate + 1-butene, +cis-2butene, + trans-2-butene, +2-methylpropene, +n-butane and +2-methylpropane. Fluid Phase Equilibria, Porvoo, v. 230, 2005, pp. $21-28$.

- SANDLER, Stanley I. et al. (Ed.). Model for thermodynamic and phase equilibria calculations. New York: Marcel Dekker, 1994. 686 p. 